\title{
Unmasking the inhibition of return phenomenon
}

\author{
SHAI DANZIGER \\ University of California, Davis, California \\ and \\ ALAN KINGSTONE \\ University of British Columbia, Vancouver, British Columbia, Canada
}

\begin{abstract}
Conventional wisdom holds that a nonpredictive peripheral cue produces a biphasic response time (RT) pattern: early facilitation at the cued location, followed by an RT delay at that location. The latter effect is called inhibition of return (IOR). In two experiments, we report that IOR occurs at a cued location far earlier than was previously thought, and that it is distinct from attentional orienting. In Experiment 1 , IOR was observed early (i.e., within $50 \mathrm{msec}$ ) at the cued location, when the cue predicted that a detection target would occur at another location. In Experiment 2, this early IOR effect was demonstrated to occur for target detection, but not for target identification. We conclude that previous failures to observe early IOR at a cued location may have been due to attention being directed to the cued location and thus "masking" IOR.
\end{abstract}

A central goal of attention research is to understand how we prepare for and select stimulus information from the environment. Typically, we attend to visual information by executing an overt shift of the head and eyes. However, attention can also be allocated covertly, without any overt movements. The effects of covert orienting on visual performance have been explored with a spatial precuing paradigm in which a response stimulus is preceded by a visual event that summons attention to a particular location (Posner, 1980, 1995; Posner, Snyder, \& Davidson, 1980). Studies using performance measures such as simple detection (Henik, Rafal, \& Rhodes, 1994; Posner, 1980), discrimination (Briand \& Klein, 1987; Jonides, 1981; Müller \& Rabbitt, 1989), and temporal order judgments (Maylor, 1985; Stelmach \& Herdman, 1991) have consistently reported more efficient processing for target stimuli appearing at a recently cued location than at noncued locations.

Both overt and covert visual orienting can be controlled exogenously or endogenously. Exogenous control refers to the external "capture" of attention by stimuli in the environment, independent of an observer's goals (Posner \& Cohen, 1984; Yantis, 1995). In contrast, endogenous control refers to an internally driven process that is charac-

This research was supported in part by NSERC Grants 52-77162 and 52-75095, and Alberta Heritage Foundation for Medical Research Grant 51-61019 awarded to A.K., and by NIH predoctoral Grant F31 MH11357-01 to S.D. and NIH Grant R01 NH 41544 to Robert Rafal. Correspondence concerning this article should be directed to $\mathrm{S}$. Danziger, School of Psychology, University of Wales, Bangor, Gwynedd, LL57 2DG, England (e-mail: s.danziger@bangor.ac.uk), or A. Kingstone, 2136 West Mall, Department of Psychology, University of British Columbia, Vancouver, BC V6T 124, Canada (e-mail: alan@cortex. psych.ubc.ca).

-Accepted by previous editor, Myron L. Braunstein terized by a strategic decision on the part of the observer to shift attention to a particular location or object in the visual scene (Jonides, 1981; Müller \& Humphreys, 1991; Warner, Juola, \& Koshino, 1990).

Research has indicated that there are empirical reasons for distinguishing between exogenous and endogenous orienting systems (see Klein, Kingstone, \& Pontefract, 1993; Rafal \& Henik, 1994; Yantis, 1995). Compared with endogenous orienting, exogenous orienting is more rapid (Cheal \& Lyon, 1991; Müller \& Findlay, 1988), difficult to inhibit (Müller \& Rabbitt, 1989), is unaffected by a concurrent task (Jonides, 1981), and is critical for the proper conjunction of stimulus features (Briand \& Klein, 1987; but see Tsal, 1989). It also appears that both forms of orienting can occur concurrently without interference (Juola, Koshino, \& Warner, 1995). Finally, there is evidence suggesting that different neural mechanisms subserve these two forms of orienting, with subcortical brain regions supporting exogenous orienting and cortical regions supporting endogenous orienting (Corbetta, Miezin, Shulman, \& Petersen, 1993; Kingstone, Grabowecky, Mangun, Valsangkar, \& Gazzaniga, 1997; Posner \& Peterson, 1990; Rafal \& Henik, 1994).

An additional difference between endogenous and exogenous covert orienting is that the latter has a biphasic effect on response time (RT) performance. That is, targets that appear at an exogenously cued location, typically in the form of a peripheral luminance increment, are initially detected more quickly than targets that appear at noncued locations; however, as cue target delays exceed several hundred milliseconds, targets appearing at a cued location are actually detected more slowly than targets appearing at noncued locations. The latter effect was coined the inhibition of return (IOR) phenomenon, reflecting the notion that RT was delayed because attention was inhibited in returning to a cued location (Posner \& Cohen, 1984). 
In light of the evidence that exogenous and endogenous orienting reflect different systems, it is important to explore the factors and conditions under which these systems may interact. After all, a moment's reflection reveals that in everyday behavior, attentional allocation is under the joint control of endogenous and exogenous orienting. For instance, before crossing the street, one first attends left and right (endogenous, voluntary orienting); but a car darting suddenly out of traffic can nevertheless capture one's attention (exogenous, reflexive orienting). Posner, Cohen, and Rafal (1982) used a paradigm that placed the two systems in competition to address whether participants can endogenously regulate exogenous orienting. In their task a peripheral cue presented in one visual field predicted that a target was likely to appear in the opposite visual field on $80 \%$ of the trials (what we will call the predicted location) with targets appearing at the stimulated location on the remaining $20 \%$ of the trials (what we will call the cued location). When the cue-target stimulus onset asynchrony (SOA) was less than $200 \mathrm{msec}$, target detection was faster at the cued location than the predicted location, even though the cue probability gave participants a strong incentive to endogenously shift attention to the predicted location in the opposite field. At SOAs exceeding $200 \mathrm{msec}$, target detection was faster at the predicted location than the cued location. These results suggested to the authors that the peripheral cue first captured attention exogenously, and then participants reoriented attention endogenously to the predicted location in the opposite field. Using the same paradigm with young and elderly individuals, Rafal and Henik (1994) found a similar pattern of results, although the facilitation effect at the predicted location occurred later for the elderly individuals. Like Posner et al. (1982), Rafal and Henik concluded that the peripheral cue first captured attention exogenously and then attention was reoriented endogenously to the predicted location, with this process of reorienting being somewhat delayed for the elderly participants.

This account, although compelling at first glance, fails to satisfy a number of questions that emerge when the data are considered closely. First, it is unclear why, in both studies, only a relatively small facilitatory effect was found at the cued location at the short SOA. That is to say, the shorter RT at the cued location relative to the predicted location was much less than what is typically found in studies using noninformative peripheral cues. This finding raises the possibility that on a significant subset of trials, participants either inhibited orienting attention to the cued location and/or successfully allocated attention to the predicted location at the earliest SOA probed. Second, given the body of evidence that exogenous and endogenous orienting are distinct, the paradigm used by these investigators does not permit an unequivocal interpretation of the response performance at the longer SOAsthat is, faster RT at the predicted location than at the cued location. One possibility, advocated by the investigators, is that attention was reallocated endogenously from the cued location to the predicted location. An al- ternative interpretation is that IOR developed at the cued location at longer SOAs, slowing detection at this location. If so, the facilitation found at the predicted location relative to the cued location may in fact reflect IOR at the cued location, voluntary orienting to the predicted location, or some unspecified combination of the two.

Warner et al. (1990) have also conducted a study in which an informative peripheral cue predicted that target onset would occur at a nonstimulated location. In this study, a cue was followed at SOAs of $0,50,100$, or $150 \mathrm{msec}$ by the appearance of a target and three distractor stimuli, each positioned at one end of an imaginary plus symbol. Participants indicated with a two-choice discrimination response whether the target was a "2" or a "5." Warner et al. found that for untrained participants, target discrimination was faster at the cued location than at the predicted location at each SOA sampled, suggesting that within the first $150 \mathrm{msec}$, only exogenous orienting was engaged.' What relationship would have emerged between exogenous and endogenous orienting if longer SOAs had been included is unknown.

In the present study we examined the nature of the interaction between endogenous and exogenous control when peripheral cues directed attention concurrently to common or distinct locations in the visual field. To accommodate the possibility that endogenous and exogenous orienting may occur in parallel (see, e.g., Juola et al., 1995), our paradigm allowed for the independent and combined effects of endogenous and exogenous orienting to be assessed across multiple target sites both for simple target detection (Experiments 1 and 2) and target identification responses (Experiment 2).

\section{EXPERIMENT 1}

There were three experimental conditions, each involving a display of four peripheral boxes positioned at $12,3,6$, and 9 o'clock. In one condition, an uninformative peripheral cue preceded target onset (the no-locationpredicted [NLP] condition). On the basis of previous research, we expected the uninformative cue to induce an exogenous shift of attention resulting in an early facilitation of target detection at the cued location replaced at the longer SOA by a delay in target detection at the cued location due to the development of IOR. The second condition was aimed at determining the effects of informative peripheral cues that predicted target onset at the cued location (the stimulated-location-predicted [SLP] condition). Working under the premise that endogenous and exogenous attentional processes operate in parallel, we expected an informative peripheral cue to induce both an endogenous and an exogenous shift of attention to the predicted/cued location. Thus, relative to the NLP condition, we predicted that rapid endogenous orienting would produce an increase in the magnitude of the facilitation effect found at the cued location at the short SOA. At the long SOA we expected some "masking" of a concurrently occurring IOR effect, since endogenous orienting to the 
cued location should shorten RT at that location and lengthen RT at noncued locations. In the third condition, a peripheral cue predicted that target onset would occur one location clockwise from the cued location (the clockwise-location-predicted [CLP] condition). The aim here was to separate the contribution of exogenous orienting to the stimulated location from the contribution of endogenous orienting based on the predictive value of the peripheral cue. We expected that performance at the cued/stimulated location relative to the unpredicted/noncued locations would reflect exogenous orienting and inhibition of return, and that performance at the predicted location relative to the unpredicted/noncued locations would reflect endogenous orienting.

In each condition, uninformative double-cue trials (e.g., the locations at 3 and 9 o'clock or 12 and $60^{\prime}$ clock might be cued together) were interspersed randomly among the single-cue trials. These double-cue trials were included as a control for a patient experiment (see Danziger, Kingstone, \& Rafal, 1998). In addition, they provide a common baseline measure across NLP, SLP, and CLP conditions.

\section{Method}

\section{Participants}

Twelve students (6 women and 6 men) ranging from 18 to 32 years of age were paid for participating in two 1.5-h sessions. All reported normal or corrected-to-normal visual acuity and were unaware of the purpose of the experiment.

\section{Materials}

Stimuli were generated on a 12 -in. Apple color monitor controlled by an LC-III Apple computer. Data collection was controlled by an external Macpacq timing apparatus that enabled millisecond accuracy in response acquisition. Participants responded by pressing a button connected to the Macpacq.

The stimulus display consisted of a black background with a gray central fixation point subtending $0.15^{\circ} \times 0.15^{\circ}$ and four gray unfilled peripheral boxes subtending $2^{\circ} \times 2^{\circ}$ positioned at $12,3,6$, and 9 o'clock, equidistant from fixation, as illustrated in Figure 1. The distance from the inner line segment of each of the four boxes to the center of the fixation point was $5^{\circ}$. The brightening (switching the outline of the peripheral boxes from gray to white) of one or two peripheral boxes ( 12 and $60^{\prime}$ clock or 3 and $90^{\prime}$ 'clock) served as the attentional cue. The target was a white cross subtending $1^{\circ} \times 1^{\circ}$ that could appear at the center of any one of the four peripheral boxes.

\section{Design}

Each of the three cue conditions was composed of a single practice block followed by a series of test blocks. In each condition, participants were to detect a target onset that was preceded by the brightening of one or two peripheral boxes (i.e., the peripheral cues). The critical difference between each condition was that the brightening of a single peripheral cue conveyed different information as to where the target might appear. In all conditions, the brightening of two peripheral cues did not provide any reliable information as to where the target might appear. The SOA between the brightening of one or two peripheral cues and target onset was either 50 or $950 \mathrm{msec}$. Single cues, double cues, and SOAs were all randomly selected. The order of conditions was counterbalanced across subjects.

In the NLP condition, the brightening of a single peripheral cue did not indicate where the target might appear. A representative practice block of 48 trials preceded four test blocks of 70 trials each.

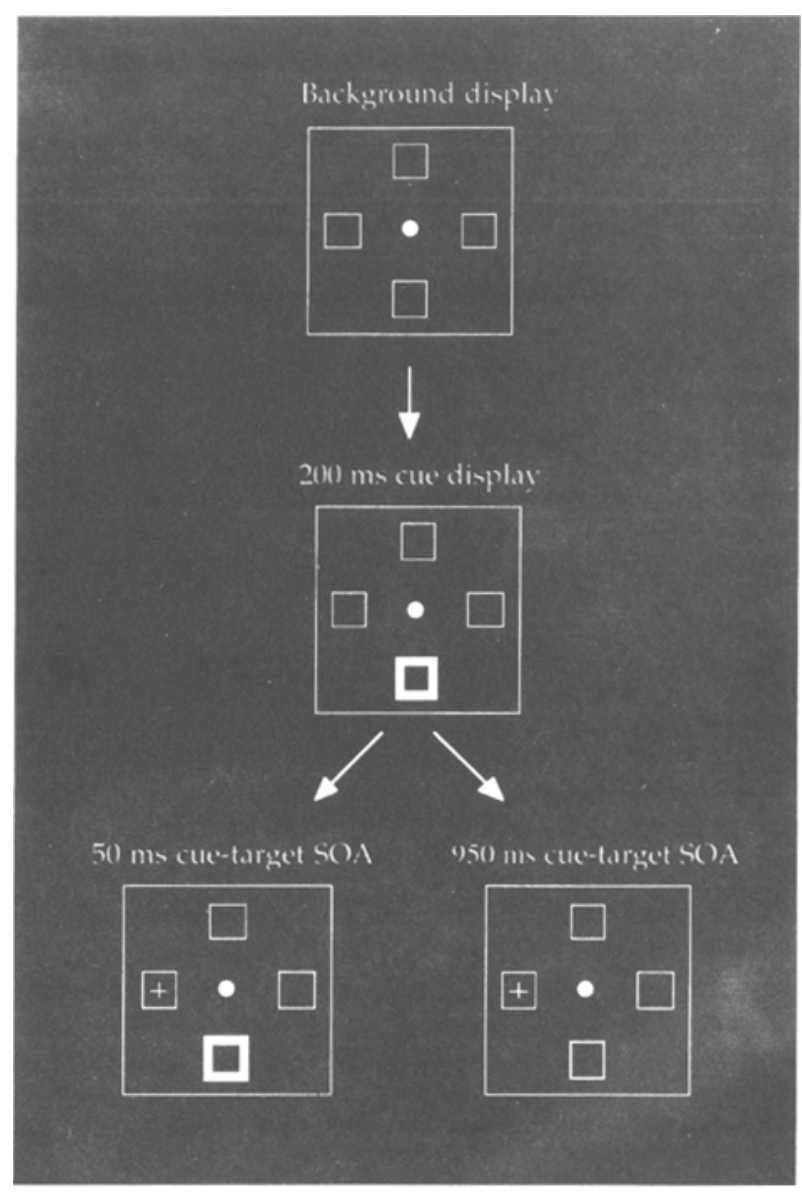

Figure 1. A representative trial sequence. A 100-msec warning tone is sounded during the initial display. A peripheral cue occurs for $200 \mathrm{msec}, 600 \mathrm{msec}$ after tone onset. Then, $50 \mathrm{msec}$ or $950 \mathrm{msec}$ after cue onset, a target cross appears at a peripheral location. In the present example, the cue at 60 'clock and the target at 90 'clock would constitute an invalid trial for the no-locationpredicted and stimulated-location predicted conditions. However, this cue-target sequence would constitute a predicted-valid trial in the clockwise-location-predicted condition.

These 280 test trials were composed of 128 single-cue trials, with the target appearing at the cued location on 32 trials and at a noncued location on 96 trials; and 128 double-cue trials, with the target appearing at a peripherally cued location on 64 trials and at a noncued location on 64 trials. In addition, there were 24 catch trials, in which a target was not presented following a single cue ( 12 trials) or double cue ( 12 trials).

In the SLP condition, single cues predicted that target onset would occur at the cued (stimulated) location $67 \%$ of the time, with target onset occurring at one of the three noncued locations $33 \%$ of the time (11\% per location). A representative practice block of 55 trials preceded six test blocks of 110 trials each. These 660 test trials were composed of 432 single-cue trials, with the target appearing at the cued location on 288 trials and at a noncued location on 144 trials; and 192 double-cue trials, with the target appearing at a peripherally cued location on 96 trials and at a noncued location on 96 trials. In addition, there were 36 catch trials ( 25 single-cue and 11 double-cue trials).

In the CLP condition, single cues predicted that target onset would occur at the location one position clockwise from the stimulated/cued 
location $67 \%$ of the time, with target onset occurring at one of the remaining three locations $33 \%$ of the time (11\% per location). For example, if the peripheral cue occurred at the 60 'clock position, as shown in Figure 1, then a target would appear $67 \%$ of the time at the 9 o'clock position and at one of the remaining three locations $33 \%$ of the time ( $11 \%$ per location). Thus, in terms of this example, the 60 'clock position is cued but not predicted; the 90 'clock position is predicted but not cued; and the 12 and 3 o'clock positions are neither predicted nor cued. A representative practice block of 55 trials preceded six test blocks of 110 trials each. These 660 test trials were composed of 432 single-cue trials, with the target appearing at the predicted location on 288 trials, at the cued location on 48 trials, and at one of the two noncued/nonpredicted locations on 96 trials; and 192 double-cue trials, with the target appearing at a peripherally cued location on 96 trials and at a noncued location on 96 trials. In addition, there were 36 catch trials ( 25 single-cue and 11 double-cue trials).

\section{Procedure}

In each of the conditions, participants were informed of the spatial relationship between the location of the single and double peripheral cues and the location of the target stimulus. They were reminded throughout the experiment to maintain central fixation. Instructions emphasized both response speed and accuracy; that is, subjects were to press the response key as quickly as possible when target onset was detected and to withhold a keypress response in all other situations.

Participants were seated $57 \mathrm{~cm}$ from the display monitor with head position maintained by a chinrest. With their preferred hand, par- ticipants made a simple keypress response when detecting a target onset at one of the four possible target locations. Rest breaks were provided between trial blocks.

At the beginning of the experimental session, the fixation point and peripheral boxes were presented. These remained present throughout the experiment. The sequence of events on each trial was as follows: An auditory tone was first sounded for $100 \mathrm{msec}$, then $600 \mathrm{msec}$ after tone onset, one or two peripheral boxes brightened for $200 \mathrm{msec}$. Target onset occurred either $50 \mathrm{msec}$ (short SOA) or $950 \mathrm{msec}$ (long SOA) after cue onset. A target remained present for $2,000 \mathrm{msec}$ or until a response was made, whichever came first. The intertrial interval was $1,500 \mathrm{msec}$. On catch trials, the warning tone signaling the start of a new trial occurred 3,500 msec after cue onset. Response latency was measured in milliseconds timed from target onset to a keypress response.

\section{Results}

Mean RTs for the single-cue data are shown in Figure 2, and those for the double-cue data are shown in Figure 3. Response anticipations (latencies less than $100 \mathrm{msec}$ ) and failures to respond in a speeded manner (latencies longer than $1,000 \mathrm{msec}$ ) were excluded from analysis.

\section{NLP Condition}

The data for both single- and double-cue trials were submitted to an analysis of variance (ANOVA) with SOA (short and long), cue validity (cued and noncued), and

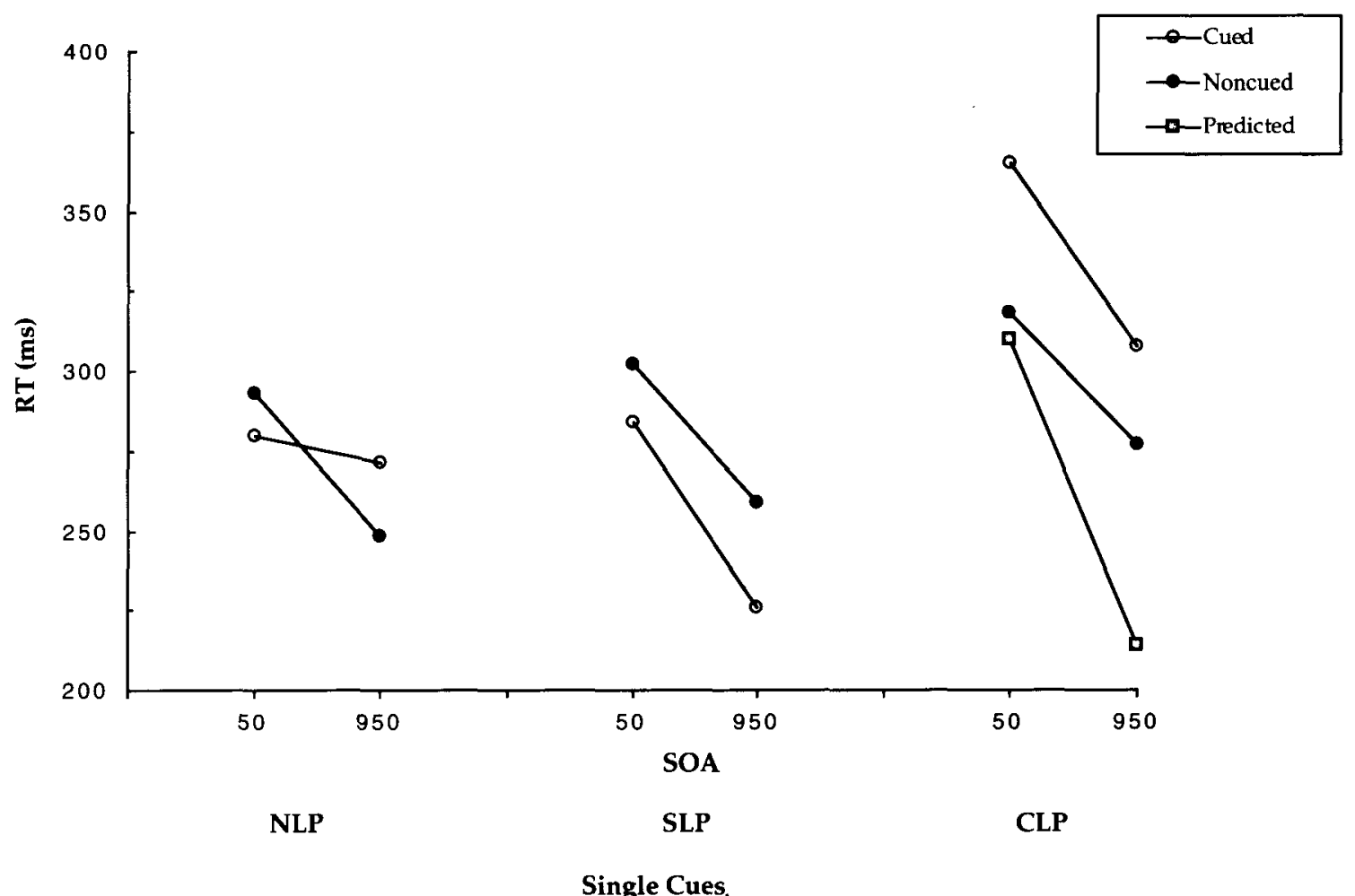

Figure 2. Mean response times (RT) in milliseconds for the single-cue data. Data are shown as a function of condition (NLP, SLP, and CLP), stimulus onset asynchrony (SOA; 50 and $950 \mathrm{msec}$ ), and cue validity (cued, noncued, and predicted noncued for the CLP condition). NLP, no location predicted; SLP, stimulated location predicted; CLP, clockwise location predicted. 


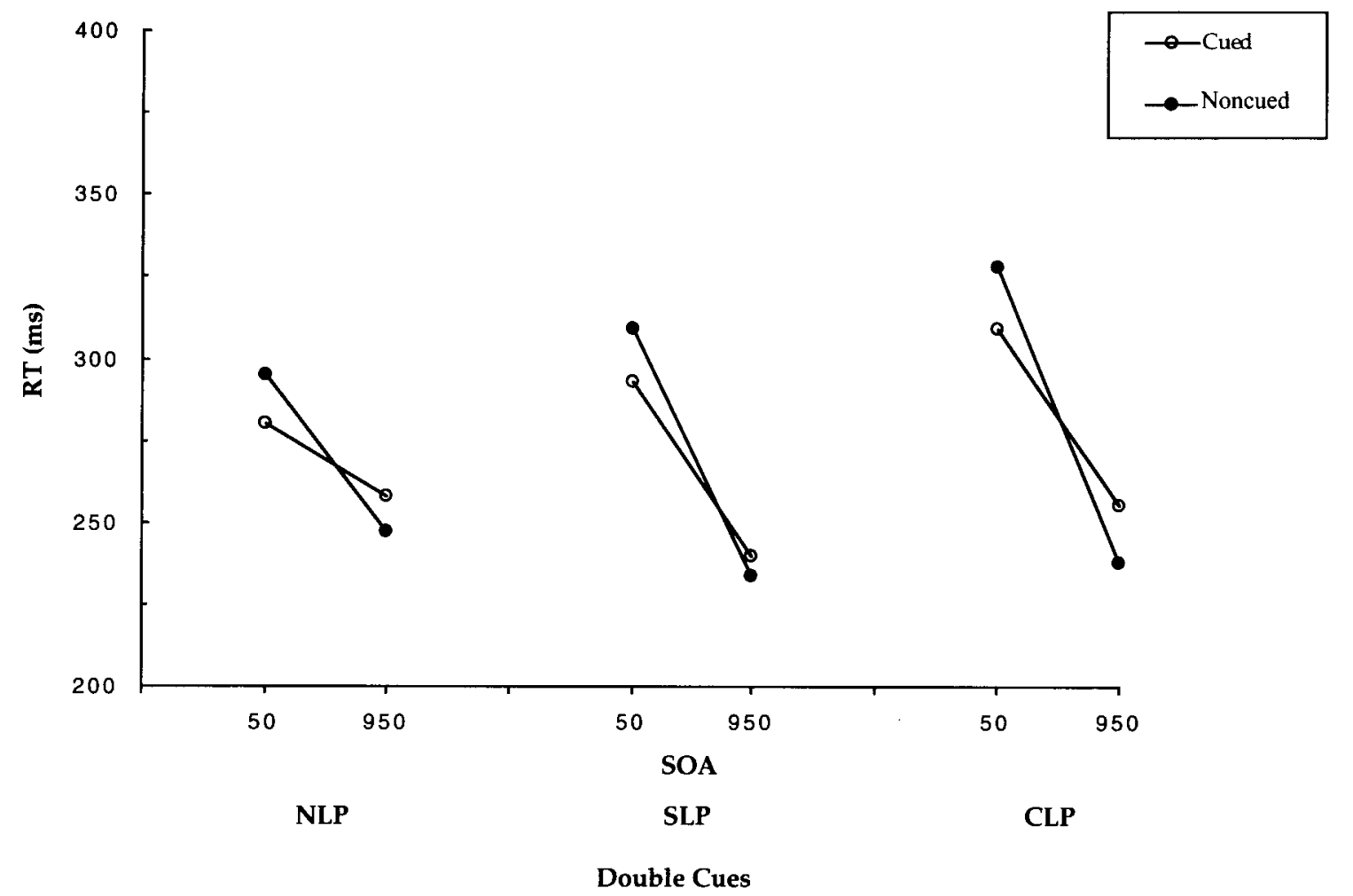

Figure 3. Mean response times (RT) in milliseconds for the double-cue data. Data are shown as a function of condition (NLP, SLP, and CLP), stimulus onset asynchrony (SOA; 50 and $950 \mathrm{msec}$ ), and cue validity (cued and noncued). NLP, no location predicted; SLP, stimulated location predicted; CLP, clockwise location predicted.

cue type (single and double) as within-subjects variables. There was a main effect of SOA, with target detection slower $(287 \mathrm{msec})$ at the short SOA than at the long SOA $[256 \mathrm{msec} ; F(1,11)=15.4, p<.005]$. SOA also interacted with cue validity $[F(1,11)=41, p<.001]$, with target detection faster at the cued location $(280 \mathrm{msec})$ than at the noncued location $(294 \mathrm{msec})$ at the short SOA $[t(11)=4.85, p<.001]$, and slower at the cued location $(264 \mathrm{msec})$ than at the noncued location $(247 \mathrm{msec})$ at the long SOA $[t(11)=3.34, p<.01]$. This early facilitation at the cued location followed by a later developing inhibition effect is the classic biphasic RT pattern associated with the IOR phenomenon. Interestingly, the SOA $X$ cue validity $X$ cue type interaction was not significant $[F(1,11)=1.1, p>.3]$, indicating that single and double cues had similar effects on response latencies.

\section{SLP Condition}

An ANOVA was conducted with the same factors as in the NLP condition. There were main effects of SOA $[F(1,11)=67.7, p<.001]$ and cue validity $[F(1,11)=$ $21.2, p<.001]$, with target detection slower at the short SOA $(297 \mathrm{msec})$ than at the long SOA $(240 \mathrm{msec})$, and faster at a cued location ( $261 \mathrm{msec}$ ) than at a noncued location $(276 \mathrm{msec})$. Cue type interacted with both SOA $[F(1,11)=7.4, p<.01]$ and cue validity $[F(1,11)=19.8$, $p<.001]$, the meaning of which is best understood within the context of the significant SOA $\times$ cue validity $\times$ cue type interaction $[F(1,11)=9.9, p<.01]$. At the short SOA, target detection was faster $[t(11)=24.81, p<.01]$ at a cued location $(289 \mathrm{msec})$ than at a noncued location $(306 \mathrm{msec})$. The cue validity $\times$ cue type interaction was not significant at this SOA $[F(1,11)=.075, p>.7]$, indicating that, as in the NLP condition, single and double cues had similar effects on target detection. In contrast, at the long SOA, the cue validity $\times$ cue type interaction was significant $[F(1,11)=16.76, p<.005]$, since target detection was faster at a cued location $(226 \mathrm{msec})$ than at a noncued location ( $259 \mathrm{msec}$ ) for single-cue trials $[t(11)=29.81, p<.001]$ but not for double-cue trials ( $240 \mathrm{msec}$ at the cued locations versus $234 \mathrm{msec}$ at the noncued locations $[t(11)=1.5, p>.15]$. The different effects of single and double cues at the long SOA suggest that following a single cue, attention was maintained endogenously at the cued location from the short to the long SOA because target onset was likely to occur at the cued location.

\section{SLP versus NLP}

To assess whether a single peripheral cue affected performance differently when it predicted target onset at the cued location than when it was spatially uninformative, we compared the single-cue data in the SLP and NLP conditions. Condition (NLP and SLP), SOA (short and 
long), and cue validity (cued and noncued) were withinsubjects factors. The key finding was a condition $\times$ SOA $X$ cue validity interaction $[F(1,11)=11.02, p<.01]$. At the short SOA, condition did not interact with cue validity $(F<1)$, indicating that the advantage at the cued location relative to the noncued location did not differ in the NLP and SLP conditions. At the long SOA, there was a significant condition $\times$ cue validity interaction $[F(1,11)=16.49, p<.005]$, reflecting the fact that in the SLP condition, target detection was significantly faster $[F(1,11)=11.98, p<.005]$ at a cued versus noncued location (a difference of $33 \mathrm{msec}$ ), whereas in the NLP condition, target detection was significantly slower $[F(1,11)$ $=9.06, p<.05]$ at a cued versus noncued location (a difference of $17 \mathrm{msec}$ ).

One question the present analysis did not address is whether IOR developed at the cued location when the cue was predictive (SLP condition). In other words, does endogenous orienting to a particular location affect the development of IOR at the cued location? The CLP condition, which was identical to the SLP condition except that a single peripheral cue predicted that target onset would occur one location clockwise from the cued location, addressed this question.

\section{CLP Condition}

Because in this condition a single peripheral cue predicted target onset at a noncued location, a target could appear at one of three different locations: the cued unpredicted location, the noncued predicted location, and the noncued unpredicted location. This unique situation required us to analyze the single and double cues separately. For single cues, the ANOVA included SOA (short and long) and cue validity (cued, predicted, and noncued) as within-subjects factors. For double cues, the ANOVA included SOA (short and long) and cue validity (cued and noncued) as within-subjects factors.

\section{Single Cues}

There was a main effect of SOA $[F(1,11)=53.9, p<$ $.001]$, with slower target detection at the short SOA $(332 \mathrm{msec})$ than at the long SOA $(266 \mathrm{msec})$; and a main effect of cue validity $[F(2,22)=24.18, p<.001]$, with the shortest RT at the predicted location $(262 \mathrm{msec})$, an intermediate RT at the noncued location $(297 \mathrm{msec})$, and the longest RT at the cued location ( $338 \mathrm{msec}$ ). Impor- tantly, the SOA $\times$ cue validity interaction was significant $[F(2,22)=10.37, p<.001]$, reflecting an increase in the advantage at the predicted location with SOA. At the short SOA, targets were detected faster at the predicted location $(311 \mathrm{msec})$ than at either a noncued location [ $318 \mathrm{msec} ; t(11)=2.23, p<.05]$ or a cued location $[365 \mathrm{msec} ; t(11)=3.46, p<.005]$. The difference in target detection at the cued and noncued locations was also significant $[t(11)=3.49, p<.005]$. At the long SOA, targets were again detected faster at the predicted location $(214 \mathrm{msec})$ than at either the noncued [ $277 \mathrm{msec} ; t(11)=$ $4.62, p<.001]$ or the cued [ $308 \mathrm{msec} ; t(11)=6.36, p<$ $.001]$ location. Again, the difference in target detection at the cued and noncued locations was significant $[t(11)=$ $4.86, p<.001]$.

\section{Double Cues}

There was a main effect of SOA $[F(1,11)=134.01$, $p<.001]$, with slower target detection at the short SOA (319 msec) than at the long SOA $(247 \mathrm{msec})$. The main finding, however, was an SOA $\times$ cue validity interaction $[F(1,11)=40.34, p<.001]$, reflecting the fact that target detection was faster at the cued location $(309 \mathrm{msec})$ than at the noncued location $(329 \mathrm{msec})$ at the short SOA $[F(1,11)=14.17, p<.005]$, and slower at the cued location $(255 \mathrm{msec})$ than at the noncued location $(239 \mathrm{msec})$ at the long SOA $[F(1,11)=14.27, p<.005]$. This is the same pattern as that observed for the double cues in the NLP and SLP conditions. ${ }^{2}$

\section{Response Accuracy}

False alarms (responses on catch trials) occurred on $2.8 \%$ of the catch trials in the NLP condition, $6.7 \%$ of the catch trials in the SLP condition, and on $5.8 \%$ of the catch trials in the CLP condition. In each of these three cue conditions, the percent of catch trial errors did not differ significantly between single and double cues.

Anticipatory responses, although rare (Table 1), were subjected to the same ANOVAs as the RT data in each of the three cue conditions. In each of the conditions there was a main effect of SOA, reflecting the fact that anticipatory responses occurred almost exclusively at the long SOA. In the SLP condition there was also a main effect of cue validity $[F(1,11)=8.51, p<.05]$, with $0.3 \%$ more anticipatory responses when the target appeared at a cued location (1.6\%) than at a noncued location (1.3\%). Fail-

Table 1

Mean Percentages of Anticipatory Responses for All Conditions

\begin{tabular}{|c|c|c|c|c|c|c|c|c|c|c|}
\hline & \multicolumn{6}{|c|}{ Single Cue } & \multicolumn{4}{|c|}{ Double Cue } \\
\hline & \multicolumn{3}{|c|}{ 50-msec SOA } & \multicolumn{3}{|c|}{ 950-msec SOA } & \multicolumn{2}{|c|}{ 50-msec SOA } & \multicolumn{2}{|c|}{ 950-msec SOA } \\
\hline & Cued & Noncued & Predicted & Cued & Noncued & Predicted & Cued & Noncued & Cued & Noncued \\
\hline NLP & 0 & 0.04 & & 2 & 1 & & 0.01 & 0 & 2 & 2 \\
\hline SLP & 0 & 0.01 & & 3.3 & 2.4 & & 0.02 & 0 & 2.8 & 2.8 \\
\hline CLP & 0 & 0 & 0 & 2.3 & 4.0 & 3.6 & 0 & 0 & 4 & 2.8 \\
\hline
\end{tabular}

Note-NLP, no location predicted; SLP, stimulated location predicted; CLP, clockwise location predicted. 
ures to respond within $1,000 \mathrm{msec}$ occurred on fewer than 10 trials in the entire experiment, with no reliable tendency to occur in any condition or SOA.

\section{Discussion}

\section{NLP Condition}

The NLP condition established the effects of uninformative peripheral cues on target detection within the context of the present paradigm. For both single and double cues, the results reflected the classic biphasic RT pattern that is typical of reflexive covert orienting: At the short SOA, target detection was facilitated at a cued versus noncued location, indicating that attention was allocated exogenously to the cued location; and at the long SOA, target detection was inhibited at a cued versus noncued location, indicating that IOR was present at the cued location. This biphasic pattern was replicated for the nonpredictive double cues across all single cue conditions (NLP, SLP, CLP), demonstrating that the different values of predictiveness attached to the single cues across conditions did not carry over to the double cues. Furthermore, these data indicate that the effect of attention was the same whether attention was oriented to a single cued nonpredictive peripheral location or to two nonpredictive cued locations (for a similar finding, see Danziger et al., 1998). From the present data, however, one cannot discriminate whether the focus of attention was divided in a noncontinuous fashion between the two cued locations (Kramer \& Hahn, 1995) or whether a single attentional "spotlight" was enlarged to encompass both locations (Posner et al., 1980). This is because target detection was not sampled at locations between those that were cued. For similar reasons, one cannot conclude from the present data whether IOR can develop independently at simultaneously stimulated locations.

\section{SLP Condition}

The SLP condition established the effects of informative peripheral cues on target detection when the cued location was also the predicted location. For single cues, target detection was faster at the cued/predicted location than at the noncued locations for both short and long SOAs. At the short SOA, the facilitation effect at the cued/ predicted location was equivalent to that in the NLP condition, suggesting that initially only exogenous attention was drawn to the cued/predicted location. An alternative interpretation is suggested in a recent study by Egly, Rafal, Henik, and Berger (in press). They found, as we did, that when the task was target detection, the early facilitatory effect of an informative peripheral cue was equivalent to that of an uninformative peripheral cue. However, when the task was target discrimination, informative peripheral cues produced greater facilitation than uninformative peripheral cues. This suggests that when the task is more attentionally demanding (e.g., perceptual resolution beyond detecting a luminance increment is required before a correct response can be executed), early endogenous orienting may facilitate performance beyond that produced solely by exogenous orienting.

At the long SOA, informative peripheral cues led to a radically different pattern of results than uninformative peripheral cues. Here, targets that appeared at a cued/predicted location were detected faster than targets appearing at noncued locations. Recall that in the NLP condition, at the long SOA, the occurrence of IOR resulted in target detection being slower at a cued location than at a noncued location. The finding of facilitation at a cued location in the SLP condition at the long SOA can therefore be attributed to endogenous orienting of attention to the cued/predicted location. What cannot be determined from the SLP data is whether IOR dwelled at the cued location despite the overall facilitatory effect found at this location. Results from the CLP condition shed light on this issue.

\section{CLP Condition}

The CLP condition established the effects of informative cues on target detection when the cued location and the predicted location were not confounded. Thus, the relationship between exogenous orienting to a peripherally cued location and endogenous orienting to a predicted location was examined when each was directed to a different spatial location. By comparing performance for single cues at the cued location, the predicted location, and the unpredicted/noncued locations, we found that target detection was shortest at the predicted location, intermediate at the unpredicted/noncued locations, and slowest at the cued location. This data pattern was observed at both the short and long cue-target SOAs. The RT advantage at the predicted location relative to the unpredicted/noncued locations increased with SOA, a finding that can be attributed to endogenous orienting to the predicted location that grows with time. ${ }^{3}$

By far the most fascinating aspect of the CLP data is the performance pattern observed at the cued location at the short SOA. In this condition, where the cued location was not the predicted location, RT was longest at the cued location. One possibility is that perceptual masking of the target by the cue contributed to this delay in RT performance. We believe, however, that such forward masking cannot account for the early inhibition effect at the cued location because identical perceptual events led to a facilitatory effect at the cued location when the cue was not spatially predictive (NLP condition) and when it predicted target onset at the cued location (SLP condition). Thus the crucial question is: What caused the early inhibition effect at the cued location? Below we discuss some possibilities.

\section{Explanations for Early Inhibition at the Cued Location in the CLP Condition}

An interaction between exogenous and endogenous orienting. On single-cue trials in the NLP and SLP conditions, attention was attracted exogenously to the cued 
location at the short SOA. The finding of an early inhibition effect at the cued location on single-cue trials in the CLP condition raises the possibility that exogenous attention was actively inhibited from being directed to the cued location - perhaps because participants had to inhibit orienting attention reflexively to the cued location in order to orient attention voluntarily to the predicted location. This notion that inhibition of exogenous orienting is a by-product of endogenous orienting is not without precedence. In the semantic priming literature, it has been shown that in order to shift from an exogenously activated category to an endogenously activated category, one actively inhibits the former (Balota, Black, \& Cheney, 1992; Neely, 1977). Similarly, when interactions between exogenous and endogenous spatial orienting have been observed, they have often been inhibitory in nature. For instance, Müller and Rabbitt (1989) showed that abrupt onsets that attract reflexive attention can interrupt voluntary processing. And perhaps more relevant to the present situation, Yantis (1995) reported that under highly focused states of voluntary spatial orienting, reflexive shifts of spatial attention to abrupt onsets can be inhibited. In the present study, if such an interaction between exogenous and endogenous spatial attention produced the early inhibition effect at the cued location, then it must have occurred very rapidly.

IOR is a separate process from endogenous and exogenous orienting. An alternative explanation is that the finding of early inhibition at the cued location reflects the leading edge of the IOR phenomenon. According to this account, IOR, exogenous attention, and endogenous attention are each separate and distinct phenomena (Posner, Walker, Friedrich, \& Rafal, 1984; Rafal, Calabresi, Brennan, \& Sciolto, 1989; see also Egly et al., in press). The finding that IOR can be produced by merely executing or preparing a saccade provides support for this hypothesis (Rafal et al., 1989), since saccade preparation alone does not trigger attentional orienting (Klein, 1980; Klein \& Pontefract, 1994).

Of course, the view that IOR and attention are separable processes begs the question as to why IOR has been observed only at long cue-target SOAs. A possible explanation is that the early IOR effect is normally masked by attentional orienting to a peripherally cued location. If this were the case, early IOR would be revealed only when attention was shifted away from the cued location, as was the case in the CLP condition.

Comparison of performance in the SLP and CLP conditions yields evidence that supports the fundamental idea that attentional orienting can mask the presence of IOR. Recall that we considered the possibility that a facilitatory effect caused by voluntary orienting to the cued location at the long SOA might be masking IOR in the SLP condition. Using the CLP condition both as a pure measure of voluntary orienting to a predicted location and as a pure measure of IOR at the cued location, we can test this possibility. In the CLP condition, the RT difference between the predicted and the unpredicted/noncued conditions was $63 \mathrm{msec}$ at the long SOA. This value is the facilitatory effect of orienting attention endogenously to the predicted location. The difference between the cued RT and the unpredicted/noncued RT was $31 \mathrm{msec}$. This is the IOR effect at the cued location at the long SOA. By combining these two values, we can calculate what the effect at the long SOA should be when the predicted and the cued location are one and the same, as was the case in the SLP condition. The combined value predicts that there should be a 32-msec facilitatory effect. Indeed, the facilitation effect in the SLP condition at the long SOA was almost exactly that: 33 msec faster at the cued location than at the noncued location.

This finding highlights two points. First, it suggests that attentional orienting and IOR can occur in parallel, with the implication being that IOR and attentional orienting are separate, isolable processes. Second, it legitimizes the possibility that attentional orienting can mask the presence of IOR (or, in principle, vice versa), supporting the notion that the early inhibition effect we found at the cued location in the CLP condition was in fact the IOR effect, which is typically masked in other paradigms by attentional orienting to the cued location.

\section{EXPERIMENT 2}

Using a precuing manipulation similar to our CLP condition, Warner et al. (1990) reported that for untrained participants, choice discrimination RT was fastest at a cued location at cue-target SOAs of 50,100, and $150 \mathrm{msec}$ (see, however, note 1). Notice that this result differs from our finding of early inhibition at the cued location in the CLP condition. Two key differences between our study and the Warner et al. investigation may account for this discrepancy.

The first difference involves the sampled range of cuetarget SOAs. Whereas we included both short and long SOAs (50 and $950 \mathrm{msec}$ ), Warner et al. (1990) sampled performance only at relatively short SOAs, ranging from 0 to $150 \mathrm{msec}$. Our CLP data indicate that although subjects can orient attention endogenously to a predicted location at a short SOA, the benefit of orienting attention to the predicted location is much smaller at a short SOA than at a long SOA. This raises the possibility that in the Warner et al. study, the cue-target SOA was so brief that it discouraged participants from orienting attention to the predicted/noncued location, and thus no inhibitory effect was observed at the cued location. Note that this explanation is consistent with both hypotheses of the early inhibition effect observed in our CLP condition. In the one case, Warner et al. did not observe an early inhibition effect because participants were not orienting attention endogenously to the predicted location, and therefore there was no inhibitory interaction between exogenous 
orienting to the cued location and endogenous orienting to the predicted location. In the other case, participants were not orienting attention endogenously to the predicted location, and therefore attentional orienting to the cued location was masking the presence of IOR.

A second distinction between the studies involves the use of different stimulus-response tasks. Whereas Warner et al. (1990) used a two-choice target identification task (with instructions to identify the target as a " 2 " or a " 5 "), we used a simple target detection task. This difference in paradigms may account for the different result patterns if the source of the effect is IOR. Several studies have shown that while IOR is obtained for target detection or target localization responses, it does not always occur for target identification responses (see Klein \& Taylor, 1994, for a recent review). Thus it possible that Warner et al.'s response task eliminated IOR, and therefore eliminated the early inhibition effect at the cued location. ${ }^{4}$ Note that this account is consistent with the hypothesis that our early inhibition effect was due to IOR, but it is not consistent with the notion that our early inhibition was due to an interaction between exogenous and endogenous processes, since there is no reason a priori to expect that a change in response task should affect this interaction.

Thus, the Warner et al. study (1990) suggested to us that we might be able to test between the two hypotheses of the early inhibition effect in the CLP condition by comparing target identification RT and target detection RT performance. If the early inhibition effect is due to IOR, then it should be present in the CLP condition when the response task is simple target detection, but it should not be present when the task is target identification. On the other hand, if the early inhibition effect is due to an inhibitory interaction between exogenous and endogenous orienting, then it should be present whether the response task is target detection or target identification.

\section{Method}

\section{Participants}

Twelve students ( 9 women and 3 men) ranging from 19 to 29 years of age were paid for participating in two 1-h sessions. All reported normal or corrected-to-normal visual acuity and were unaware of the purpose of the experiment.

\begin{abstract}
Materials
Materials were the same as Experiment 1, with the following exceptions. Stimuli were presented on a 17 -in. Apple color monitor. The target stimulus was an upright or inverted uppercase letter $\mathrm{T}$ that subtended $1^{\circ}$ in height and $0.75^{\circ}$ in width. And finaliy, the stimulus background was light gray and the cues and targets were black. This final change controlled for the possibility that in Experiment 1 forward masking of the target by a luminant cue contributed to the early inhibition effect in the CLP condition, although, as previously discussed, forward masking most likely did not play a role because identical perceptual events occurred in the NLP and SLP conditions, in which facilitatory effects were observed at the cued location.
\end{abstract}

\section{Design}

The design was identical to that of Experiment 1 save for the following changes. First, only single cues were presented. The trial count for the simple target detection task was the same as in Ex- periment 1 minus the double-cue trials. The trial count for the identification RT task was equivalent to that of the target detection count minus the catch trials. Second, each observer participated in only the NLP and CLP conditions, once with two-choice identification RT as the dependent measure and once with simple target detection RT as the dependent measure. In light of recent evidence that the IOR effect may decline with practice (Weaver, Lupiànez, \& Watson, 1998), we ran the NLP condition before the CLP condition, with response condition counterbalanced across participants. This provided a conservative test of the hypothesis that the early inhibition effect in the CLP condition was due to IOR.

\section{Procedure}

The procedure was identical to that of Experiment 1 except for the following four changes. Eye movements were monitored throughout the experiment by an experimenter viewing a video camera. The cue duration was shortened from 200 to $100 \mathrm{msec}$ to further reduce possible masking effects of the cue on the target. To compile a more detailed time course of the effects in the CLP condition, there were three cue-target SOAs: 50, 450, and $850 \mathrm{msec}$. And finally, in the target identification task, participants pressed the left button of the button box when an upright letter $T$ was presented and the right button when an inverted letter $T$ was presented. Orientation varied randomly, and equiprobably, from tria! to trial.

\section{Results}

Data handling was the same as in Experiment 1. Mean RTs for the simple detection data are shown in Figure 4 and those for the target identification data are shown in Figure 5. Response accuracy data are shown in Table 2.

\section{Target Detection RT}

NLP condition. The data were submitted to an ANOVA with SOA (short, medium, and long) and cue validity (cued and noncued) as within-subjects variables. There were main effects of SOA $[F(2,22)=4.91, p<.05]$, and cue validity $[F(1,11)=15.97, p<.005]$, with target detection slowest at the short SOA (314 msec), intermediate at the middle SOA $(289 \mathrm{msec})$, and fastest at the long SOA $(256 \mathrm{msec})$; and slower at the cued location (304 msec) than at the noncued location $(296 \mathrm{msec})$. SOA interacted with cue validity $[F(2,22)=29.9, p<.001]$. Target detection was faster at the cued location $(303 \mathrm{msec})$ than at the noncued location $(324 \mathrm{msec})$ at the short SOA [t(11) $=$ $3.96, p<.005]$, slower at the cued location $(299 \mathrm{msec})$ than at the noncued location $(279 \mathrm{msec})$ at the medium SOA $[t(11)=4.92, p<.001]$, and slower at the cued location (309 msec) than at the noncued location $(284 \mathrm{msec})$ at the long SOA $[t(11)=7.7, p<.001]$. These results replicate those of Experiment 1 , indicating that with a noninformative peripheral cue and a target detection response task, RT is facilitated at the short SOA, with the IOR effect appearing at longer SOAs.

CLP condition. The data were submitted to an ANOVA with SOA (short, medium, and long) and cue validity (cued, predicted, and noncued) as within-subjects factors. There were main effects of SOA $[F(2,22)=20.17$, $p<.001]$ and cue validity $[F(2,22)=55, p<.001]$. Target detection was slowest at the short SOA ( $341 \mathrm{msec})$, intermediate at the long SOA (304 msec), and fastest at the medium SOA ( $289 \mathrm{msec})$; and target detection was slow- 


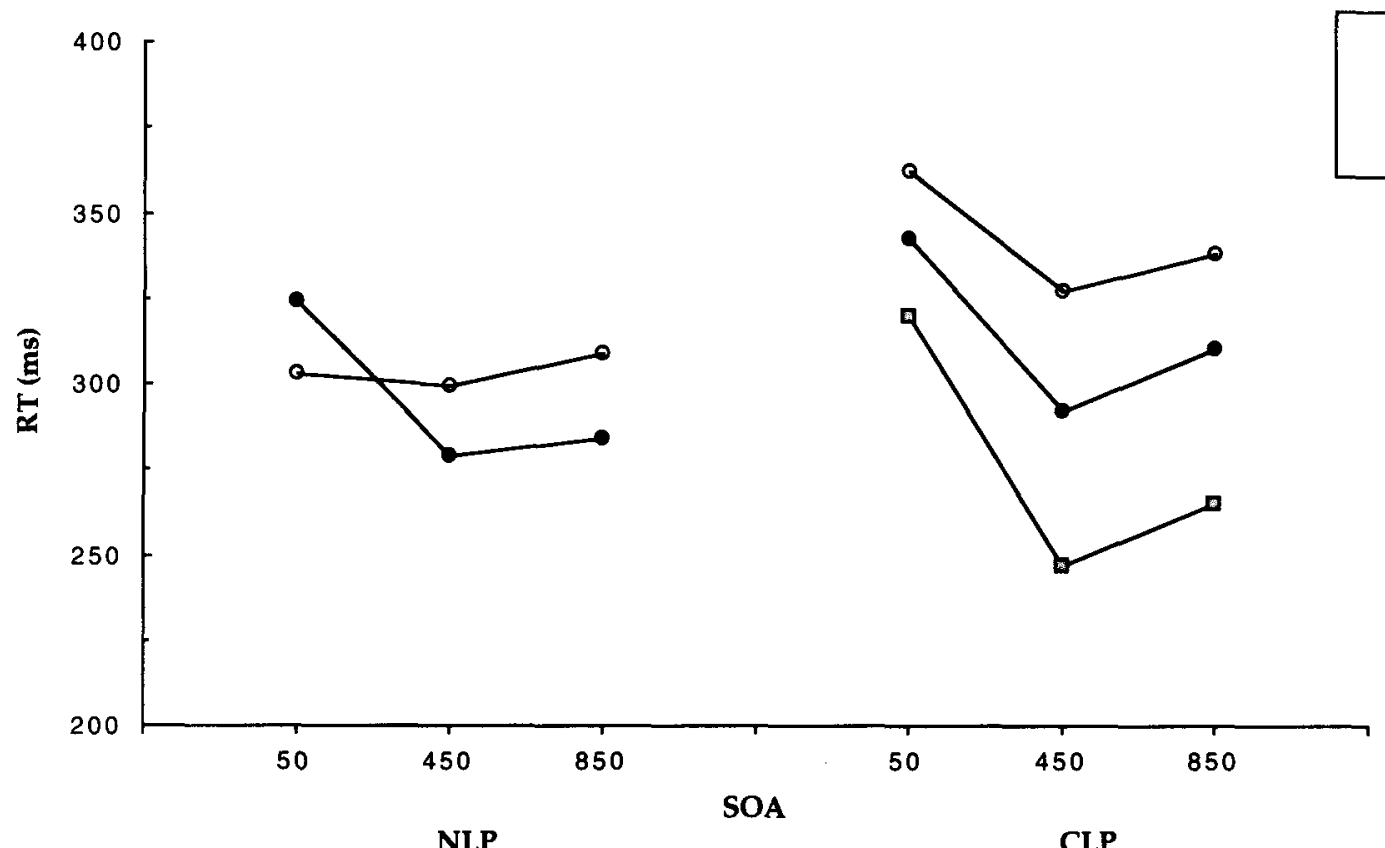

Simple Target Detection

Figure 4. Mean response times (RT) in milliseconds for the simple target detection data. Data are shown as a function of condition (NLP and CLP), stimulus onset asynchrony (SOA; 50, 450, and 850 msec), and cue validity (cued, noncued, and predicted noncued for the CLP condition). NLP, no location predicted; CLP, clockwise location predicted.

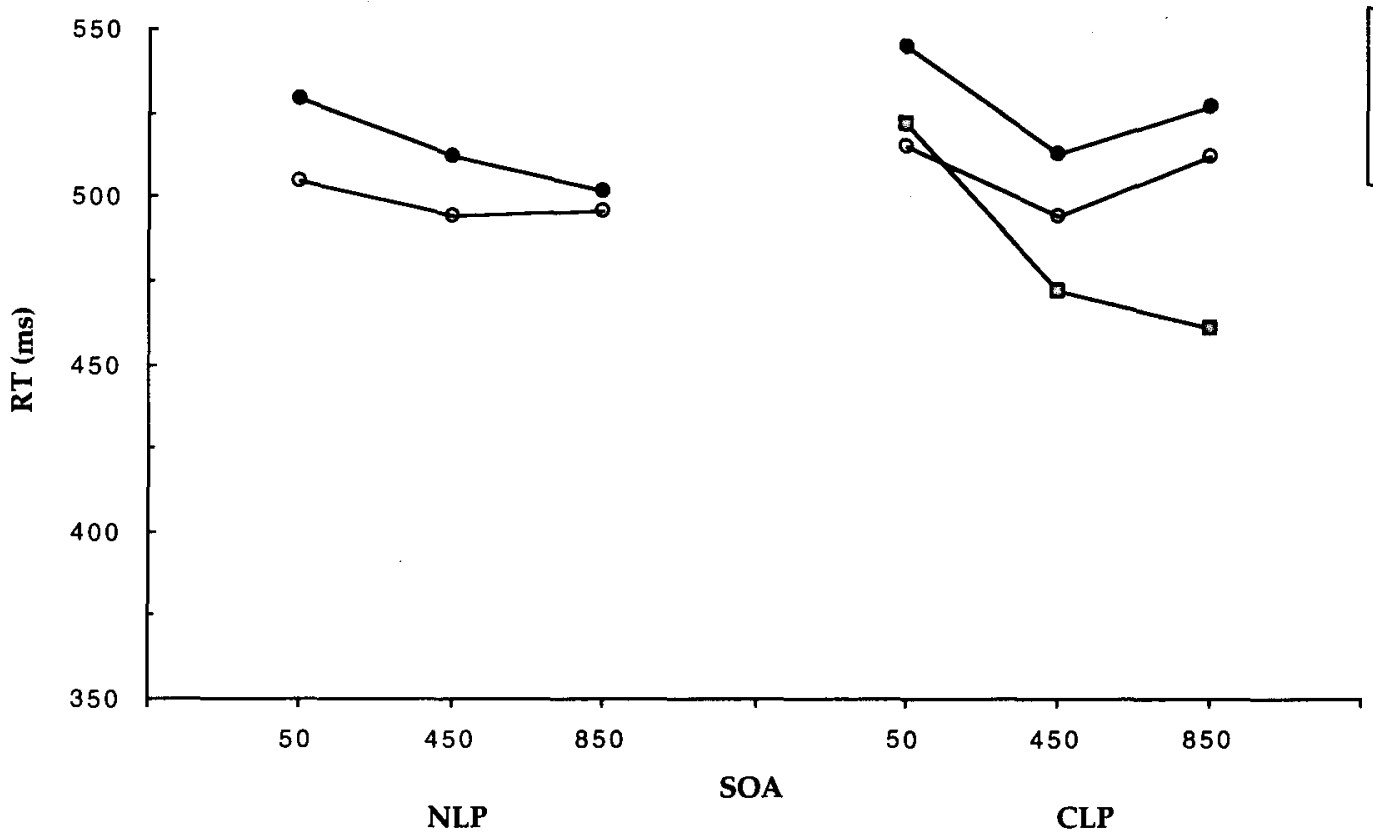

$$
\begin{aligned}
& \rightarrow \text { Cued } \\
& \rightarrow-\text { Noncued } \\
& \rightarrow-\text { Predicted }
\end{aligned}
$$

\section{Choice Discrimination}

Figure 5. Mean response times (RT) in milliseconds for the target identification data. Data are shown as a function of condition (NLP and CLP), stimulus onset asynchrony (SOA; 50, 450, and $850 \mathrm{msec}$ ), and cue validity (cued, noncued, and predicted noncued for the CLP condition). NLP, no location predicted; CLP, clockwise location predicted. 
est at the cued location ( $342 \mathrm{msec}$ ), intermediate at the noncued location ( $315 \mathrm{msec})$, and fastest at the predicted location $(277 \mathrm{msec})$. Importantly, the SOA $\times$ cue validity interaction was significant $[F(4,44)=5.29, p<.005]$. This interaction resulted from smaller differences in RT between the predicted location, the cued location, and the noncued location at the short SOA than at either the medium or long SOAs. However, at each of these SOAs, target detection was significantly faster at the predicted location than at the noncued location, and was significantly faster at the noncued location than at the cued location (all $t \mathrm{~s}<.005$ ). These results replicate those of the CLP condition in Experiment 1.

\section{Target Identification RT}

NLP condition. An ANOVA was conducted with SOA (short, medium, and long) and cue validity (cued and noncued) as within-subjects variables. There was a main effect of cue validity $[F(1,11)=25.4, p<.001]$, with target identification faster at the cued location $(498 \mathrm{msec})$ than at the noncued location $(514 \mathrm{msec})$. There were no other significant effects. Thus, in contrast to the results from the simple detection task, only a facilitatory effect was observed at the cued location; that is, there was no evidence of IOR.

CLP condition. The ANOVA was conducted with SOA (short, medium, and long) and cue validity (cued, predicted, and noncued) as within-subjects factors. There were main effects of SOA $[F(2,22)=13.26, p<.001]$ and cue validity $[F(2,22)=11.25, p<.001]$, with target identification slower at the short SOA $(528 \mathrm{msec})$ than at the medium (493 $\mathrm{msec})$ and long $(500 \mathrm{msec}) \mathrm{SOAs}$; it was slowest at the noncued location $(528 \mathrm{msec})$, intermediate at the cued location $(507 \mathrm{msec})$, and fastest at the predicted location $(485 \mathrm{msec})$. The SOA $\times$ cue validity interaction was also significant $[F(4,44)=6.3, p<$ $.001]$. At the short SOA, targets were identified faster at the predicted location [522 msec; $t(11)=2.97, p<.05$ ] and at the cued location [ $515 \mathrm{msec} ; t(11)=2.73, p<.05$ ] than at the noncued location $(545 \mathrm{msec})$. There was no RT difference between the predicted and cued locations $[t(11)=0.577, p>.5]$. At the medium SOA, targets were identified more quickly at the predicted location $(472 \mathrm{msec})$ than at the noncued location [513 msec; $t(11)=3.35$, $p<.01]$. The difference between the cued $(494 \mathrm{msec})$ and the noncued location fell just short of significance $[t(11)=$ $1.86, p<.09$ ], and there was no significant difference between the cued and the predicted locations $[t(11)=$ $1.57, p>.1]$. Finally, at the long SOA, targets were identified faster at the predicted location $(461 \mathrm{msec})$ than at either the cued location [ $512 \mathrm{msec} ; t(11)=4.87, p<.001]$ or the noncued locations [527 msec; $t(11)=4.82, p<$ $.001]$. There were no other significant differences.

Response accuracy. In the simple target detection conditions, false alarms (responses on catch trials) occurred on $2.5 \%$ of the catch trials in the NLP condition and on $3.5 \%$ of the catch trials in the CLP condition. Anticipatory responses (Table 2) and erroneous responses in the target identification conditions (participants pressed a button representing an upright letter $T$ when in fact the target was an inverted letter $T$, and vice versa) were subjected to the same ANOVAs as the RT data.

Anticipatory responses (target detection). Anticipatory responses accounted for $0.4 \%$ of the trials in the NLP condition and $1.3 \%$ of the trials in the CLP condition. There were no significant effects in the NLP condition. In the CLP condition, the only significant effect was of SOA, $[F(2,22)=10.31, p<.001]$, with more anticipatory responses at the medium SOA $(2.8 \%)$ than at either the short SOA $(0.2 \%)$ or the long SOA $(0.7 \%)$.

Anticipatory responses and selection errors (target identification). Anticipatory responses accounted for $0.1 \%$ of the trials in the NLP condition and $2 \%$ of the trials in the CLP condition. There were no significant effects in the NLP condition. In the CLP condition, the only significant effect was of SOA $[F(2,22)=4.28, p<.05]$, with more anticipatory responses at the short SOA (3.1\%) than at either the medium SOA (1.5\%) or the long SOA (1.5\%).

Selection errors accounted for $2.4 \%$ of the data in the NLP condition and $4 \%$ of the trials in the CLP condition. In the NLP condition, there was a main effect of cue validity $[F(1,11)=8.45, p<.05]$, with more errors made when a target appeared at a noncued location $(3.3 \%)$ than at a cued location (1.5\%). In the CLP condition, there was

Table 2

Mean Percentages of Anticipatory Responses (Simple Target Detection and Target Identification) and Errors (Target Identification) for All Conditions

\begin{tabular}{|c|c|c|c|c|c|c|c|c|c|}
\hline & \multicolumn{3}{|c|}{ 50-msec SOA } & \multicolumn{3}{|c|}{ 450-msec SOA } & \multicolumn{3}{|c|}{ 850-msec SOA } \\
\hline & Cued & Noncued & Predicted & Cued & Noncued & Predicted & Cued & Noncued & Predicted \\
\hline \multicolumn{10}{|c|}{ Simple Target Detection } \\
\hline NLP & 0 & 0.35 & & 0 & 0.7 & & 1 & 0.3 & \\
\hline CLP & 0.5 & 0 & 0.1 & 2.6 & 3.1 & 2.8 & 0 & 1.5 & 0.6 \\
\hline \multicolumn{10}{|c|}{ Choice Discrimination } \\
\hline NLP & $0 / 0.5$ & $0.1 / 4$ & & $0.5 / 1.5$ & $0.1 / 3$ & & $0 / 2.6$ & $0.1 / 2.4$ & \\
\hline CLP & $2.6 / 4.7$ & $4.8 / 6.8$ & $2 / 4.7$ & $1.7 / 3.6$ & $2.4 / 5.4$ & $0.5 / 2.2$ & $1.1 / 3.1$ & $2.7 / 3.6$ & $0.4 / 2$ \\
\hline
\end{tabular}

Note-For the target identification data, the values to the left of the " " symbol are the anticipation percentages and those to the right are the response selection errors. NLP, no location predicted; CLP, clockwise location predicted. 
a main effect of SOA $[F(2,22)=3.88, p<.05]$, with more errors at the short SOA (5.4\%) than at either the medium SOA $(3.8 \%)$ or the long SOA $(3 \%)$.

\section{Discussion}

\section{Target Detection}

The results of the NLP and CLP conditions replicate and extend those reported in Experiment 1. In the NLP condition, target detection was faster at the cued location than the noncued location at the shortest SOA, with IOR appearing at the cued location at the two longer SOAs. In the CLP condition, target detection was fastest at the predicted location, intermediate at the unpredicted/noncued locations, and slowest at the cued location at each of the three SOAs. The RT advantage at the predicted location relative to the unpredicted/noncued locations grew from the short SOA $(20 \mathrm{msec})$ to the medium SOA (45 $\mathrm{msec}$ ) and then remained constant, whereas the inhibition at the cued location relative to the unpredicted/noncued locations remained constant across SOAs in the range of 20 to $30 \mathrm{msec}$. These data are consistent with the view that endogenous orienting to the predicted location increases with time, while the inhibition effect present at the cued location appears rapidly and is sustained across the SOAs sampled.

\section{Target Identification}

The results in the target identification task differed dramatically from those of the target detection task. In the NLP condition, target identification was faster at the cued location than at the noncued location at each of the three SOAs. Thus in the NLP condition, there was evidence for facilitation at the cued location at the short SOA, but no evidence for IOR at the cued location at the longer SOAs. In the CLP condition, at the short SOA target identification RT was faster at the cued and predicted locations than at the unpredicted/noncued locations. There was no significant difference between the cued and predicted RTs. At the intermediate SOA, the RT advantage at the predicted location grew relative to both the cued and the unpredicted/noncued locations, but again was significantly faster than only the unpredicted/noncued locations. The 19-msec RT advantage at the cued location relative to the unpredicted/noncued locations fell just short of significance. Finally, at the long SOA, the RT advantage at the predicted location continued to grow and was significantly faster than both the cued and the unpredicted/ noncued locations. The $15-\mathrm{msec} \mathrm{RT}$ advantage at the cued location relative to the unpredicted/noncued locations was not significant.

The NLP and CLP data demonstrate that inhibition effects did not develop at the cued location when target identification was required. The effects observed in the CLP data at the short SOA $(50 \mathrm{msec})$ are similar to the 50-msec SOA data reported in the Warner et al. (1990; Experiment 1) study in that there was no inhibition at the cued location. They differ however, in that we found a significant RT advantage at the predicted location relative to the noncued location, whereas Warner et al. did not (see, however, note 1). One possibility for this discrepancy is that the target in our study was presented in isolation, whereas in the Warner et al. experiment, target onset coincided with the appearance of a distractor at each of the other three locations. These abrupt exogenous onsets at the other locations may have interfered with the endogenous allocation of attention to the predicted location. Whatever the case may be, the present target identification data do not support the notion that the inhibition effect results from competition between endogenous and exogenous orienting, because if it did, one would expect for it to be found whenever attention was to be redirected from a peripherally cued location, independent of response mode.

\section{Failures to Find Early Inhibition in Previous Target Detection Studies}

Two other studies that required simple target detection and that used precuing procedures similar to those of the present study failed to find an early inhibition effect at the cued location in a precuing condition equivalent to our CLP condition (Posner et al., 1982; Rafal \& Henik, 1994). We, however, have speculated that the data for these studies may be consistent with the presence of an inhibition effect at the cued location. This speculation is based on the fact that in both studies the facilitatory effect at the cued location was small, indicating that an inhibition effect and a facilitatory effect may have co-occured. The present findings support this interpretation. Why Posner et al. (1982) and Rafal and Henik still found residual facilitation at the cued location at the short SOA is not clear, but may have to do with any number of differences between their study and the present investigation, including differences in the cue-target SOAs used and differences in the number of possible target locations (they had only two, whereas we had four locations). The important point is not why their findings and our findings are not precisely the same, but that our findings strongly suggest that Posner et al. (1982) and Rafal and Henik found the same basic effect that we have found, which is an early inhibition at a cued location when this cued location predicted target onset elsewhere.

\section{Conclusion}

Previous studies have produced convincing evidence that exogenous and endogenous covert orienting are qualitatively different processes. Exogenous orienting is rapid and short lived. Endogenous orienting is relatively slower and sustained. Our experiments are consistent with these views. The new finding is that when attention is directed away from a cued location to a predicted location, and the task is target detection, an early, and sustained, inhibitory effect is observed at the cued location. We tested whether this inhibitory effect reflects IOR or an inhibitory interaction between exogenous and endogenous 
orienting. The results suggest that the early inhibitory effect reflects IOR. It is our conclusion that IOR occurs early at a peripheral location, and that IOR is a process that is distinct from exogenous or endogenous orienting. Previous failures to observe an early IOR effect at the cued location may have been due to attention being directed initially to the cued location, thus masking the presence of IOR.

\section{REFERENCES}

Balota, D. A., Black, S. R., \& Cheney, M. (1992). Automatic and attentional priming in young and older adults: Reevaluation of the twoprocess model. Journal of Experimental Psychlogy: Human Perception \& Performance, 18, 485-502.

Briand, K. A., \& KLein, R. M. (1987). Is Posner's "beam" the same as Treisman's "glue"? On the relation between visual orienting and feature integration theory. Journal of Experimental Psychology: Human Perception \& Performance, 13, 228-241.

Cheal, M. L., \& Lyon, D. R. (1991). Central and peripheral cuing of forced-choice discrimination. The Quarterly Journal of Experimental Psychology, 43A, 859-880.

Corbetta, M., Miezin, F., Shulman, G. L., \& Petersen, S. E. (1993). A PET study of visuospatial attention. Journal of Neuroscience, 13, 1202-1226.

Danziger, S., Kingstone, A., \& Rafal, R. (1998). Orienting to extinguished signals in hemispatial neglect. Psychological Science, 9, 119-123.

Egly, R., Rafal, R. D., HeniK, A., \& Berger, A. (in press). Reflexive and voluntary covert orienting in detection and discrimination tasks. Journal of Experimental Psychology: Human Perception \& Performance.

Henik, A., Rafal, R. D., \& Rhodes, D. (1994). Endogenously generated and visually guided saccades after lesions of the human frontal eye fields. Journal of Cognitive Neuroscience, 6, 400-411.

JoNIDES, J. (1981). Voluntary versus automatic control over the mind's eye's movement. In J. B. Long \& A. D. Baddeley (Eds.), Attention and performance $I X$ (pp. 187-203). Hillsdale, NJ: Erlbaum.

Juola, J. F., Koshino, H., \& Warner, C. B. (1995). Tradeoffs between attentional effects of spatial cues and abrupt onsets. Perception \& Psychophysics, 57, 333-342.

Kingstone, A., Grabowecky, M., Mangun, G. R., Valsangkar, M., \& Gazzaniga, M. S. (1997). Paying attention to the brain. The study of selective visual attention in cognitive neuroscience. In J. Burak \& J. Enns (Eds.), Attention, development, and psychopathology (pp. 263287). New York: Guilford Press.

Kingstone, A., \& Pratt, J. (in press). Inhibition of return is composed of attentional and oculomotor processes. Perception \& Psychophysics.

KLEIN, R. M. (1980). Does oculomotor readiness mediate cognitive control of visual attention? In R. S. Nickerson (Ed.), Attention and Performance VIII (pp. 259-275). Hillsdale, NJ: Erlbaum.

Klein, R. M., Kingstone, A., \& Pontefract, A. (1993). Orienting of visual attention. In K. Rayner (Ed.), Eye movements and visual cognition: Scene perception and reading (pp. 46-65). New York: SpringerVerlag.

Klein, R. M., \& Pontefract, A. (1994). Does oculomotor readiness mediate cognitive control of visual attention? Revisited! In C. Umiltà \& M. Moscovitch (Eds.), Attention and performance XV: Conscious and nonconscious information processing (pp. 333-350). Cambridge, MA: MIT Press, Bradford Books.

Klein, R. M., \& TAYLOR, T. L. (1994). Categories of cognitive inhibition with reference to attention. In D. Dagenbach \& T. H. Carr (Eds.), Inhibitory process in attention, memory, and language (pp. 113-150). San Diego, Academic Press.

Kramer, A. F., \& HAHN, S. (1995). Splitting the beam: Distribution of attention over non-contiguous regions of the visual field. Psychological Science, 6, 381-386.

MAYLOR, E. A. (1985). Facilitatory and inhibitory components of ori- enting in visual space. In M. I. Posner \& O. S. M. Marin (Eds.), $A t$ tention and performance $X I$ (pp. 189-204). Hillsdale, NJ: Erlbaum. Müller, H. J., \& FindLAY, J. M. (1988). The effect of visual attention on peripheral discrimination thresholds in single and multiple element displays. Acta Psychologica, 69, 129-155.

MüLler, H. J., \& HumphreYs, G. W. (1991). Luminance-increment detection: Capacity-limited or not? Journal of Experimental Psychology: Human Perception \& Performance, 17, 107-124.

Müller, H. J., \& Rabbitt, P. M. A. (1989). Reflexive and voluntary orienting of attention: Time course of activation and resistance to interruption. Journal of Experimental Psychology: Human Perception \& Performance, 15, 315-330.

NeELy, J. H. (1977). Semantic priming and retrieval from lexical memory: Roles of inhibitionless spreading activation and limited-capacity attention. Journal of Experimental Psychology: General, 106, 226254.

Posner, M. I. (1980). Orienting of attention. Quarterly Journal of Experimental Psychology, 32, 3-25.

PoSNer, M. I. (1995). Attention in cognitive neuroscience: An overview. In M. S. Gazzaniga (Ed.), The cognitive neurosciences (pp. 615-624). Cambridge, MA: MIT Press.

POSNER, M. I., \& COHEN, Y. P. C. (1984). Components of visual orienting. In H. Bouma \& D. Bouwhuis (Eds.), Attention and performance X: Control of language processes (pp. 531-556). Hillsdale, NJ: Erlbaum.

Posner, M. I., Cohen, Y. P. C., \& Rafal, R. D. (1982). Neural systems control of spatial orienting. Philosophical Transactions of the Royal Society of London: Series B, 298, 187-198.

Posner, M. I., \& PETERSON, S. (1990). The attention system of the human brain. Annual Review of Neuroscience, 13, 25-42.

Posner, M. I., SNyder, C. R. R., \& Davidson, B. J. (1980). Attention and the detection of signals. Journal of Experimental Psychology: General, 109, 160-174.

Posner, M. I., Walker, J. A., Friedrich, F. J., \& Rafal, R. D. (1984). Effects of parietal injury on covert orienting of visual attention. Journal of Neuroscience, 4, 1863-1874.

Pratt, J., Kingstone, A., \& KhoE, W. (1997). Inhibition of return in location- and identity-based choice decision tasks. Perception \& Psychophysics, 59, 964-971.

Rafal, R. D., Calabresi, P., Brennan, C., \& Sciolto, T. (1989). Saccade preparation inhibits reorienting to recently attended locations. Journal of Experimental Psychology: Human Perception \& Performance, 15, 673-685.

RAFAL, R. D., \& HENIK, A. (1994). The neurology of inhibition. In T. Carr \& D. Dagenbach (Eds.), Inhibitory processes in attention, memory and language (pp. 1-51). New York: Academic Press.

Stelmach, L. B., \& Herdman, C. M. (1991). Directed attention and perception of temporal order. Journal of Experimental Psychology: Human Perception \& Performance, 17, 539-550.

TSAL, Y. (1989). Further comments on feature integration: A reply to Briand and Klein. Journal of Experimental Psychology: Human Perception \& Performance, 15, 407-410.

WARNer, C. B., Juola, J. F., \& Koshino H. (1990). Voluntary allocation versus automatic capture of visual attention. Perception \& Psychophysics, 48, 243-251.

WEAVEr, B., LuPiáñez, J., \& Watson, F. L. (1998). The effects of practice on object-based, location-based, and static-display inhibition of return. Perception \& Psychophysics, 60, 993-1003.

YANTIS, S. (1995). Attentional capture in vision. In A. F. Kramer, M. G. H. Coles, \& G. D. Logan (Eds.), Converging operations in the study of visual selective attention (pp. 45-76). Washington: American Psychological Association.

\section{NOTES}

1. In a second experiment that examined the effect of practice on spatial orienting, Warner et al. (1990) found that for trained participants, target discrimination was faster at the predicted location than the cued location at each SOA sampled, with no difference in RT between the cued and noncued locations, suggesting that subjects could circumvent automatic attentional capture. 
2. An ANOVA of the double cues was conducted with condition (NLP, SLP, and CLP), SOA, and cue validity as within-subjects factors. There was no effect of condition, although there was an SOA $\times$ cue validity interaction $[F(1,11)=90.19, p<.001]$, indicating that the facilitation effect at the short SOA and the IOR effect at the long SOA did not differ significantly between conditions.

3. This finding perhaps provides the strongest evidence for the view that the early facilitation at the cued location was equivalent in the SLP and NLP conditions because of a ceiling effect. The alternative, that endogenous orienting to the cued/predicted location was too slow in the SLP condition, can be rejected given our present finding that voluntary orienting to the predicted-yet noncued-location resulted in early facilitation.

4. At present there is no agreed upon account as to why IOR has been found consistently for target detection responses but has not been found consistently for target identification responses. Klein and Taylor (1994) have suggested that this may be because IOR is actually more closely associated with response inhibition than attentional inhibition, as suggested by others (e.g., Posner \& Cohen, 1984). According to Klein and Taylor, peripheral cues activate spatially directed motor responses, which within the context of the cuing paradigm must be inhibited because a target has not yet appeared. If a target then appears at the cued location, demanding the execution of the inhibited spatial response, IOR is observed. Target identification responses that are not based on the location of the target should therefore not produce IOR (see Kingstone \& Pratt, in press; Pratt, Kingstone, \& Khoe, 1997). At present, a potential weakness of the Klein and Taylor account is that it hinges on the untested asssumption that detection responses are based implicitly on the location of a target stimulus.

(Manuscript received November 25, 1996; revision accepted for publication July 2,1998 .) 